\title{
Expressed sequence tags from heat-shocked seagrass Zostera noltii (Hornemann) from its southern distribution range
}

\author{
Sónia I. Massa ${ }^{a}$, Gareth A. Pearson ${ }^{a}$, Tânia Aires ${ }^{a}$, Michael Kube ${ }^{b}$, Jeanine L. Olsen ${ }^{c}$,
} Richard Reinhardt ${ }^{b}$, Ester A. Serrão ${ }^{a}$, Sophie Arnaud-Haond ${ }^{d, *}$

\footnotetext{
${ }^{a}$ CCMAR-CIMAR, Universidade do Algarve, Gambelas; 8005-139 Faro; Portugal

${ }^{b}$ Max-Planck Institute for Molecular Genetics, Innestraße 63/73; 14195 Berlin; Germany

${ }^{\mathrm{c}}$ Department of Marine Benthic Ecology and Evolution, Centre for Ecological and Evolutionary Studies, Biological Centre, University of Groningen, 9750 AA Haren; The Netherlands

d IFREMER, Centre de Brest, BP 70, 29280 Plouzané; France
}

\author{
*: Corresponding author: Sophie Arnaud-Haond: Tel.: + 332982243 05; fax: + 33298224757 ; \\ email address : Sophie.Arnaud@ifremer.fr
}

\begin{abstract}
:
Predicted global climate change threatens the distributional ranges of species worldwide. We identified genes expressed in the intertidal seagrass Zostera noltii during recovery from a simulated low tide heat-shock exposure. Five Expressed Sequence Tag (EST) libraries were compared, corresponding to four recovery times following sub-lethal temperature stress, and a non-stressed control. We sequenced and analyzed 7009 sequence reads from $30 \mathrm{~min}, 2 \mathrm{~h}, 4 \mathrm{~h}$ and $24 \mathrm{~h}$ after the beginning of the heat-shock (AHS), and 1585 from the control library, for a total of 8594 sequence reads. Among 51 Tentative UniGenes (TUGs) exhibiting significantly different expression between libraries, 19 (37.3\%) were identified as 'molecular chaperones' and were over-expressed following heat-shock, while 12 (23.5\%) were 'photosynthesis TUGs' generally under-expressed in heat-shocked plants. A time course analysis of expression showed a rapid increase in expression of the molecular chaperone class, most of which were heat-shock proteins; which increased from 2 sequence reads in the control library to almost 230 in the 30 min AHS library, followed by a slow decrease during further recovery. In contrast, 'photosynthesis TUGs' were under-expressed 30 min AHS compared with the control library, and declined progressively with recovery time in the stress libraries, with a total of 29 sequence reads $24 \mathrm{~h}$ AHS, compared with 125 in the control. A total of 4734 TUGs were screened for EST-Single Sequence Repeats (EST-SSRs) and 86 microsatellites were identified.
\end{abstract}

\section{Highlights}

Response to heat stress is very fast but gene expression returns to normal after $24 \mathrm{~h}$. Photosynthesis-related genes were under-expressed after heat-shock. Heat-shock caused a quick rise in heat shock proteins and molecular chaperone expression.

Keywords : EST library ; High temperature stress ; Abiotic stress response ; Climate change 


\section{Introduction}

Seagrasses are key-species in coastal ecosystems, as important primary producers providing food, nursery and shelter for many marine species, but are declining worldwide $[1,2]$; changes attributed to anthropogenic activities and climate change $[1$, 3]. The dwarf seagrass Zostera noltii dominates the intertidal habitats of the coastal lagoon system Ria Formosa, in southern Portugal, where at low tide this species occurs either in shallow intertidal pools or completely emersed. The predicted increase in sea surface temperatures (SST) of $0.2^{\circ} \mathrm{C} /$ decade [4] is raising concerns as to the ability of this species to survive the expected global warming, particularly since intertidal habitats are more affected by air temperature than SST. Previous work suggested that the current temperature in shallow intertidal pools is already very close to the temperature tolerance of approximately $38^{\circ} \mathrm{C}$ determined for Z. noltii in the Ria Formosa [5], with records showing temperatures as high as $36^{\circ} \mathrm{C}$ in the summer of 2007. Plants that occur in the upper intertidal can be exposed for as long as 6 hours during spring tides, and temperature in these small intertidal pools have reached $38^{\circ} \mathrm{C}$ in low wind/high sun and air temperature situations (S. Massa, personal observation). When higher plants are exposed to temperatures higher than required for optimal growth, a cellular adaptive response is activated to maintain cellular homeostasis under stress, resulting in increased synthesis of heat-shock proteins (HSP) and reduced production of other metabolic proteins [6]. Heat-shock can affect macromolecular synthesis, levels of cations, states of protein phosphorylation, metabolic pathways and cytoskeleton networks [7]. Heat stress has been shown to reduce photosynthetic yield in tropical seagrasses and increase photoinhibition [8]. It also reduces chlorophyll levels, reducing light (energy) absorption in chloroplasts for photosynthesis, and affects the levels of protective enzymes against oxidative stress, such as ascorbate peroxidase 
(APX) and superoxide dismutase (SOD) $[9,10]$. All of this suggests that the response to

71 heat stress is a complex process involving a large number of genes.

As genomic tools become more accessible for a wide range of non-model

organisms, they can be applied by evolutionary ecologists to address questions

concerning adaptation to novel or stressful environments, in order to clarify whether changes in the phenotype of independent populations confronted with similar environmental challenges are adaptive or a result of random genetic drift [11-13].

Differences in individual responses to adverse conditions affect fitness through selection, and may ultimately influence the survival of populations and potential shifts in species distribution ranges [14].

As of February $5^{\text {th }}, 2011$ there were 13,434 marine seagrass EST sequences reads in NCBI, from only 2 species, of which 10,345 are from the closely related species Zostera marina and 3,089 from Posidonia oceanica, and none for Z. noltii. This study aims to identify genetic markers and genes expressed in the intertidal seagrass $Z$. noltii during recovery from heat-shock exposure, simulating a low-tide event. A description of transcriptional changes in Z. noltii in response to heat-shock is needed to study the impacts of temperature stress and to explore the adaptation potential of this species in the face of climate change. (e.g. molecular markers including SSR and SNPs to identify loci potentially under selection, and gene sequence information to develop qPCR assays for functional studies of target genes). Moreover, an increased coverage of the transcriptome of seagrasses in general will allow the identification of crucial clusters

91 of genes systematically involved in response to warming, providing a basis for comparative studies of gene evolution in seagrasses. 


\subsection{Culture conditions and stress treatment}

96

Z. noltii plants were collected in the spring of 2007 at 4 sites in the Ria Formosa

97 (Ramalhete, Praia de Faro, Olhão and Portimão) in several distant cores with natural sediment, and placed in an outdoor tank $(1.5 \times 1.5 \mathrm{~m}$, maximum seawater volume 225 L) with ambient light conditions, continuous seawater flow from the Ria Formosa and simulated tides for acclimation during approx. 4 weeks. In high tide situation, water level in the tank was up to its maximum capacity, and every day at 10 a.m. the water level was slowly decreased until roughly two hours later only a $2 \mathrm{~cm}$ layer of water (approximately) remained above the sediment, mimicking a low tide situation. At 4 p.m., ambient seawater was gradually added to increase the water level back to high tide situation. This process was repeated again starting at 10 p.m. as tides in the Ria Formosa are semidiurnal. Previous work had determined the sub-lethal temperature for Z. noltii shoots in Ria Formosa to be slightly above $37^{\circ} \mathrm{C}$ [5], and so a single heat-shock of $37.5 \pm 0.5^{\circ} \mathrm{C}$ was then applied for four hours, the approximate average duration of low tide exposure in this part of Z.noltii distributional range, during a simulated low tide situation as previously described, between 10 am and $2 \mathrm{pm}$. After the heat-shock, ambient seawater was gradually added to the tank to lower the temperature to its initial value of approx. $22^{\circ} \mathrm{C}$. Sampling of inner leaves of randomly picked shoots (on average, $59 \mathrm{x}$ approx. 20 shoots per time step) from all sites and cores for RNA extraction occurred at 30 minutes, 2, 4 and 24 hours after the beginning of the heat-shock. Each group of approximately 20 shoots was put in a tube and immediately frozen in liquid nitrogen for later processing. A sample of non-stressed plants was also collected to be used as a control (blank).

\subsection{RNA preservation and storage}


At the end of the first three samplings (plus the control), samples were taken to the lab

121 in liquid nitrogen and immediately freeze-dried for at least 48 hours, and preserved at $80^{\circ} \mathrm{C}$ until extraction. The same procedure was performed after the 24 hour sampling.

\section{3. cDNA library construction}

125 Samples were transferred into a tube with a tungsten sphere and ground at $30 \mathrm{~g}$ for 10 minutes. RNA extraction was performed using Qiagen and GE Healthcare extraction kits (Germany). RNA quality was verified using $3 \mu \mathrm{L}$ of RNA extraction on denaturing agarose gels and quantification was performed with a spectrophotometer at 260 and 280 nm.

RNA extractions were treated with the Macherey-Nagel NucleoSpin RNAII kit for DNAse I digestion. All cDNA libraries were constructed with purified mRNA using Dynabeads ${ }^{\circledR}$ mRNA purification kit (Invitrogen); each sample was fractionated by column chromatography and the highest quality fractions were pooled together and directionally cloned into pDORN 222, using CloneMiner ${ }^{\mathrm{TM}}$ cDNA Library Construction Kit (Invitrogen). Electrocompetent cells were transformed by electroporation and then grown in SOC medium. After plasmid extraction, sequence data were obtained using M13-21 primer (5'-TGTAAAACGACGGCCACT-3'), amplified by the following programme: denaturation step $\left(96^{\circ} \mathrm{C} 1^{\prime}\right)$, followed by 35 cycles of denaturation $\left(96^{\circ} \mathrm{C}\right.$ $\left.20^{\prime \prime}\right)$, annealing $\left(55^{\circ} \mathrm{C} 10^{\prime \prime}\right)$ and elongation $\left(60^{\circ} \mathrm{C} 4^{\prime}\right)$. The cloned cDNAs were $5^{\prime}$-endsequenced using Big Dye 3.1 chemistry and ABI 3130XL capillary sequencers.

\subsection{Sequence processing and EST assembling}

143 Each library was treated independently to allow comparison of the response to stress over time. An average of 1,719 clones were sequenced for each of the five treatments 
145 (30 min, 2h, 4h, 24h and control) and analysed with the ESTragon program (developed 146 and routinely used in the Max Planck Institute for Molecular Genetics) for removal of 147 low quality [15] and/or contaminated sequence reads from each dataset before 148 alignment, with a cut-off length of $100 \mathrm{bp}$. All vector-clipped and high quality sequence 149 reads were submitted to dbEST within GenBank [accession numbers HO214335150 HO215643 for the control library, HO215644-HO217278 for 30 minutes, HO217279151 HO218949 for 2 hours, HO218950-HO220485 for 4 hours and HO220486-HO222020 for 24 hours]. Processed sequence reads were then assembled into contigs to cluster the individual sequence reads to represent unique transcripts using TGICL [16]. Unique sequences, either singletons or contigs, will from now on be referred to as Tentative UniGenes (TUGs). Blastx analysis was performed with Blast2GO® (V 2.4.4) $[17,18]$ against NCBI non redundant (nr) protein database, using a preliminary E-value of $10^{-3}$ as cutoff, to maximise the number of annotation terms obtained. Blastx results were then mapped and annotated against the Gene Ontology (GO) database, according to three different categories: Biological Process (BP), Molecular Function (MF) and Cellular Component (CC). Annotation parameters were as follows: E-value Hit filter

$1611.0 \mathrm{E}^{-6}$, annotation cutoff 55, GO Weight 5. Fisher's Exact Tests were performed to test for significance of differential expression in each heat-shock library versus the control library.

\subsection{Homology searching}

Individual stress libraries were screened by local Blastn against a database consisting of a pooled assembly of all stress treatment libraries (i.e., $30 \mathrm{~min}, 2 \mathrm{~h}, 4 \mathrm{~h}$ stress, and $24 \mathrm{~h}$ recovery) to identify homologues and estimate their expression levels. In this case, when two or more TUGs from individual stress libraries had the same top blast hit 
against this database, they were considered the same TUG. In the case of the control

171 library that was not included in the database, we applied a stringent E-value cut-off of $<$

$1.0 \mathrm{E}^{-100}$ when considering homologous consensus TUG sequences.

TUGs with significantly different expression levels over the time-course of the

stress treatment, or between stressed and non-stressed samples, were identified with the

IDEG6 (Identification of Differentially Expressed Genes) web tool [19], using the

Audic and Claverie method [20] to compare, after normalization, the number of sequence reads/TUG in each library (significant threshold of $\alpha=0.05$, corrected for multiple testing with $[(\mathrm{m}+1) / 2 \mathrm{~m}])$. Only TUGs with a total of at least 10 sequence reads from all libraries were used in the expression analysis, in an attempt to control for random sequencing effects in small TUGs. The analysis resulted in a total of 51 TUGs with significantly different expression in at least one pairwise library comparison. These were then screened using Blastn against Dr. Zompo's EST databases for Z. marina and $P$. oceanica to find interspecific matches $[21,22]$ with an bit score cutoff value of 200 .

\subsection{Microsatellite identification}

All TUGs from all 5 libraries were screened for single sequence repeats (EST-SSRs) or microsatellites using MsatCommander [23], for all motifs with two to six nucleotides length and a minimum of six repeat units.

\section{Results and Discussion}

\subsection{EST sequencing determination}

192 A total of 8,594 clones from all libraries were sequenced, resulting in 7,799 high quality sequence reads. After screening for cloning relics and contamination, a total of 113 reads were removed, leaving a total of 7,686 successful reads. Clustering (TGICL) 
resulted in 170 contigs for the 30 min library, 174 contigs for the $2 \mathrm{~h}$ library, 151 contigs for the $4 \mathrm{~h}$ library, and 145 contigs for both the $24 \mathrm{~h}$ and the control libraries. Average percentage of sequence reads with a successful blast against NCBI non redundant (nr) protein database was $82 \%$, of successfully mapped sequences $70 \%$ and successfully annotated against the GO database was $56 \%$. Detailed information for each library can 200 be found in table 1. The main protein families found in our data were the HSP70 protein family, the protein kinase domain family and the chlorophyll a-b binding protein family (Table 2). The reduction in the number of sequence reads of the $H S P 70$ protein family between the stress and the control libraries was very clear, while the number of chlorophyll a-b binding proteins was roughly the same among libraries.

\subsection{Identification of differential expression}

Out of the 51 TUGs with significantly different expression between libraries and a total of at least 10 sequence reads, $26(51 \%)$ had a matching hit with Z. marina, 16 (31\%) of which also had a match with P. oceanica (Annex I). Most of the highest-scoring matches were photosynthesis-related TUGs but also included 2 heat-shock cognate protein 80 TUGs, a stress-induced phosphoprotein, a glyceraldehyde-3-phosphate dehydrogenase, a flavoprotein, a $70 \mathrm{kDa}$ peptidyl-prolyl isomerase, a glutamine

214 synthetase and one TUG with no significant Blastx hit, suggesting that these TUGs are

215 highly conserved across the seagrass species, probably due to their fundamental role in 216 cellular pathways. The remaining 13 TUGs (25\%) had no meaningful matches. These 21751 TUGs were divided into 3 groups according to their function: 'molecular 218 chaperones', 'photosynthesis TUGs' and 'other TUGs of interest', which will be 219 discussed further below. 


\subsection{Molecular chaperones}

222

Among 943 TUGs from the control library, only two heat-shock protein TUGs were

223

found, and 61 heat-shock protein TUGs were found in the combined stress libraries,

224 from a total of 4,666 TUGs. When the number of sequence reads was taken into consideration, IDEG found 9 of these heat-shock protein TUGs to have a significantly different expression between libraries. 'Molecular chaperones' include not only these 9 heat-shock protein TUGs but also 10 other TUGs, like heat-shock cognate proteins and

DnaJ homologues, and were over-expressed in the stress libraries. While the control library showed only 2 sequence reads, these increased rapidly at the beginning of the stress, showing a total of 229 sequence reads at 30 minutes, and slowly decreased thereafter to 213 at 2 hours, 126 at 4 hours and 66 at 24 hours (Fig. 1). The response was very fast, with significant difference being recorded as soon as 30 minutes after the beginning of the heat-shock.

A total of eight sequences ( 5 contigs and 3 singletons) encoding small heat shock proteins ( $(H S P s)$ were identified in our dataset based on pfam database searches of predicted ORFs. ORF prediction was performed using the OrfPredictor tool [24] available at http://proteomics.ysu.edu/tools/OrfPredictor.html. The resulting ORFs were used to identify Pfam domains on the UFO webserver (http://ufo.gobics.de/submission, [25]). All 8 sequences had start sites and encoded a predicted full-length $s H S P$ with an HSP20/alpha crystalline domain (PF00011). In two separate cases a singleton and

241 contig were found to have identical protein sequences, but variable 3' UTRs. The other

2423 contigs and one singleton encoded unique proteins. Searches for signal peptides

243 targeting the proteins to the chloroplast or mitochondrion were negative, and no

244 transmembrane domains were identified, suggesting that these proteins are putatively 
cytoplastic. Heat-shock proteins are highly conserved among species; their main role is to protect and repair protein structures, preventing the denaturation process or promoting the proper refolding of denatured proteins [7]. Since protein conformation is also important in normal conditions, most HSPs are present in all cells and tissues even in the absence of stress factors such as elevated temperatures [26]. Heat-shock proteins are also involved in the apoptotic pathways, as irreparably damaged cells will eventually be eliminated when stress conditions are too severe [27]. Previous work has shown that mortality will eventually occur (Massa et al, unpubl.). After 24 hours the response appeared to be complete as Fisher's Exact Test showed no further differences between $24 \mathrm{~h}$ expression and the control library (Annex 2), suggesting a return to normal gene expression 24 hours after the heat-shock. proteins, which stimulate the ATPase activity of $H S P 70$ protein [28], that catalyse the folding of proteins and the assembly of protein complexes $[29,30]$ and heat-shock cognate (Hsc) 70 and 80 (Fig 1). Although HS cognates are constitutively expressed, it has been shown that $H S C 70$ is required for Heat-Shock Factor 1 to become activated and target expression of appropriate genes during heat stress [31]. Molecular chaperones are important during both unstressed and stressful conditions, as they not only assist in the de-novo folding of denatured proteins but also in conformational changes that affect function, while they also transport unfolded proteins across membranes and plasmodesmata $[32,33]$. The stress-induced phosphoprotein 1 , that we also included in this group of 'molecular chaperones', was first described in of some $H S P 70$ genes [34]. 
270 [21] where no over-expression of heat-shock proteins was observed in the EST library

271 constructed for elevated growth temperature. However, this may reflect the differences

272 in the organismal biology and ecology of this species in comparison with intertidal $Z$.

273 noltii (the Z. marina populations used were subtidal and therefore exist in an

274 environment with considerably lower amplitudes and extreme values of temperature

275 fluctuation). The major aim of the Z. marina study was to investigate sub-lethal growth

276 temperature $\left(25^{\circ} \mathrm{C} ; 2^{\circ} \mathrm{C}\right.$ higher than an expected summer maximum) rather than heat

277 shock per se. If a minor heat-shock response was induced, it may have subsided by the

278 time of sampling, as our results show that the response may be over between 4 and $24 \mathrm{~h}$

279 after the end of the shock.

280 Reusch and colleagues suggested that the lack of heat-shock protein expression

281 in Z. marina might be explained by the fact that permanently submerged seagrasses, as

282 was the case with Z. marina in their experiments, are seldom subject to rapid

283 temperature fluctuations because of the 'buffered' aquatic environment, lending contrast

284 to terrestrial environments where the presence of heat-shock proteins genes is a

285 common response to temperature stress [21]. Our results demonstrate that for intertidal

286 marine angiosperms like Z. noltii, the geographic distribution of which extends to

287 warmer latitudes where Z. marina is strictly subtidal, temperature in small intertidal

288 pools is indeed more influenced by air than by sea temperatures; the former reach levels

289 very close to the sub-lethal temperature of $Z$. noltii shoots during summer in the Ria

290 Formosa [5]. The regular and rapid increase of temperature in small intertidal pools,

291 together with potential desiccation, may therefore require the maintenance of a heat

292 stress response in dwarf eelgrass more similar to terrestrial angiosperms than subtidal

293 ones. 


\subsection{Photosynthesis TUGs}

296 We found 12 TUGs with photosynthetic function and with significantly different expression between libraries, which were overall under-expressed in the stress libraries. The control library showed a total of 125 sequence reads, which decreased to 96 after 30 min, 86 after 2h, 44 after $4 \mathrm{~h}$, and reached a minimum of 29 after 24h (Fig. 2). Most of the TUGs encode for chlorophyll a-b binding proteins that transfer energy between photosystem antennae and reaction centers, and suggests a general down-regulation of photosynthetic activity. This is consistent with physiological studies showing that the photosynthetic yield of $Z$. noltii decreases immediately after a heat-shock [5, 35]. The photosynthetic machinery is integrated and composed of many subunits making it energetically expensive for the cell to produce under stress [30]. Therefore, the downregulation of TUGs related to photosynthetic components under heat-shock, such as highly abundant chlorophyll a-b binding proteins, could reveal a trade-off that helps maintain energy balance. However, since previous work has shown that about half of the plants will eventually die following a similar heat-shock [5], under-expression of photosynthesis TUGs may also be a consequence of apoptosis. regulation after $2 \mathrm{~h}$ of a CP24 protein (ELIP/psbS family; Figure 2). Members of this protein family are thought to be important in non-photochemical quenching of excess light energy and protection against photo-oxidative stress [36].

\subsection{Other TUGs of interest}

317 A number of other TUGs with diverse functions were also found to have significantly different expressions among libraries (Fig. 3). 
Ubiquitin

321 Ubiquitin is a highly conserved small protein, involved in the selective degradation of many short-lived proteins in eukaryotic cells as they are targeted for degradation by covalent ligation to ubiquitin. Ubiquitin-mediated degradation of regulatory proteins

324 plays important roles in the control of numerous processes, including cell-cycle progression, signal transduction, transcriptional regulation, receptor down-regulation, and endocytosis. The ubiquitin system has been implicated in the immune response, development, and programmed cell death $[37,38]$. Ubiquitin TUGs were overexpressed in the stress libraries, suggesting that heat-stress may have damaged some proteins, which were targeted for proteolysis.

Glutamine synthetase

Another TUG that was also slightly elevated in the stress libraries was glutamine synthetase (GS), involved in the assimilation of nitrogen and a biomarker of plant metabolism, indicating nutrient deficiency under stress conditions [39]. Higher plant GS in roots functions in the primary assimilation of ammonia from the soil. In leaves, GS is also responsible for the reassimilation and detoxification of the large amounts of ammonia lost during photorespiration [40]. Accumulation of nitrogen may be explained by the lack of use of this nutrient for growth during stress conditions, as was observed in Z. marina during reduced light conditions [41].

341 Response to oxidative stress and/or cellular detoxification

342 The classical plant peroxidases are a well-studied group of heme-containing enzymes

343 that utilize either $\mathrm{H}_{2} \mathrm{O}_{2}$ or $\mathrm{O}_{2}$ to oxidize a wide variety of substrates [42]. In the majority

344 of plant species investigated they occur as distinctive isoenzymes which can be 
constitutive or induced in response to external factors such as wounding, stress and attack by pathogens [43]. Peroxidase showed an over-expression in the middle of the heat-shock (2 hours) when compared to the remaining libraries. catalyse a number of distinct glutathione-dependent reactions: in addition to their ability to catalyse the formation of conjugates, GST can also serve as peroxidases and isomerases. They play a critical role in protecting against electrophiles and products of oxidative stress and cellular detoxification generally, suggesting that they are part of an adaptive response to chemical stress [44]. This TUG was also over-expressed in the stress libraries, suggesting that GST may also participate in the response to temperature stress.

Plant metallothioneins are involved in heavy metal tolerance and detoxification and might also be involved in regulation of cellular availability of required heavy metals, namely copper and zinc $[45,46]$ and were under-expressed in the stress libraries, suggesting cell energy may be switched from heavy metal detoxification to heat stress response .

Reticulon-like protein

Reticulons are proteins that have been found predominantly associated with the endoplasmic reticulum in yeast and mammalian cells. Although reticulon-like proteins have been identified in plants, very little is known about their cellular localization and functions, but have been shown to be associated with the endoplasmic reticulum in Arabidopsis thaliana [47]. Reticulon-like proteins showed a slight variation among stress libraries, being under-expressed during the beginning of the heat-shock (30 
minutes and 2 hours libraries), suggesting a decrease in a hypothetical housekeeping gene during high stress conditions.

Peptidyl-prolyl isomerase

373 Peptidyl prolyl isomerases are protein folding catalysts $[48,49]$, whose function is the 374 cis-trans isomerization of peptidyl-prolyl bonds, a relevant conformational change that 375 is rate limiting. Most, but not all, peptide bonds are connected in the trans conformation 376 during biosynthesis at the ribosomes, and this conformation is also found in the native 377 structure of most peptide bonds [50]. The over-expression of these TUGs in the stress 378 libraries may suggest an increase in protein biosynthesis, which is possibly related to 379 the production of stress-response molecules such as heat-shock proteins.

381 Isoflavone reductase homolog

382 Isoflavone reductase (IFR) is an enzyme specific to isoflavonoid biosynthesis, a 383 pathway which is mainly found in the Leguminosae (angiosperms). It catalyses a

384 NADPH-dependent reduction involved in the biosynthesis of important and related 385 phenylpropanoid-derived plant defense compounds [51]. Interestingly, this enzyme was over-expressed in the 24 hours library, which may reflect a delayed response to the stress.

Flavoprotein WrbA

390 The flavoprotein WrbA, originally described as a tryptophan $(W)$ repressor- $b$ inding 391 protein in Escherichia coli, has recently been shown to exhibit the enzymatic activity of 392 a NADH: quinone oxidoreductase to maintain of a supply of reduced quinine, and having a possible role in stress response [52]. WrBA was under-expressed in the first 
stress library (30 minutes), which suggests this enzyme might be inhibited by high temperatures.

396

397

398

Glyceraldehyde-3-phosphate dehydrogenase

Glyceraldehyde 3-phosphate dehydrogenase $(G A P D H)$ is a nucleic-acid-binding protein involved in glycolysis and in the Calvin cycle [53-55] and was under-expressed under stress conditions, suggesting an inhibition of important metabolic pathways during high temperature stress. GAPDH exhibits high temperature sensitivity [56] and the inhibition of this enzyme affects the photosynthetic process, by causing a secondary photoinhibitory response in PSII [57] that may be the cause of the decrease in photosynthetic activity. It can also reduce energy generation if glycolysis is inhibited and ATP levels decrease.

407

\subsection{Microsatellite identification}

409 A total of 4,734 TUGs were screened for EST-SSRs and 86 microsatellites were 410 identified, with 43 di-, 36 tri-, 4 tetra- and 3 hexanucleotides (Table 3). The main 411 advantage of EST-SSR over SSR markers developed by random genomic screening is 412 their physical linkage to coding sequences. In future studies, these may be used to 413 identify genomic regions associated with heat stress response, as markers can be 414 designed around a SSR in a candidate gene. Another advantage is their apparently high 415 conservation between species and even genera, probably because these are more likely 416 to be in gene-rich regions of chromosomes, which can facilitate the screening of related 417 species even when no EST resources are available [58]. 


\section{Conclusions}

420 This study reports the first transcriptomic dataset for Z. noltii, focusing on response to

421 high temperature stress. Understanding the molecular basis of traits of interest has been

422 hindered by a lack of genomic resources for this species, and this study has provided a

423 considerable dataset covering the transcriptional response to heat-stress. Almost 8,600

424 sequences reads were produced from all libraries, which resulted in over 3,000

425 annotated TUGs. As expected, an important part of the TUGs with significantly

426 different expressions between libraries were known to be stress-related, mostly heat-

427 shock proteins, which were over-expressed in stress libraries and are commonly induced

428 by high temperature stress. Also, photosynthetic activity seems to be affected by heat-

429 shock, as important enzymes are thermally inhibited and cells redirect their energy

430 towards defense strategies. The heat-shock response in Z. noltii is very fast, showing

431 significant differences from unstressed plants as soon as 30 minutes after the beginning

432 of the stress, causing an increase of heat-shock protein TUGs and an inhibition of some

433 of the TUGs with photosynthetic function. A total of 86 microsatellites were also

434 identified, and in future work may be used to develop genetic markers in candidates

435 TUGs related to the response to heat stress.

437 5. Acknowledgments:

438 This research was funded by the project DIVSTAB (POCI/MAR/60179/2004) of the

439 Portuguese Science Foundation FCT, FEDER, the Marine Genomics Network of

440 Excellence (MGE GOCE-CT-2003-505446) and a fellowship from FCT and ESF (to

441 SM). We thank Sven Klages and Cymon Cox for their assistance in the data analysis

442 and João Reis and Miguel Viegas for their technical assistance in the setup of

443 experimental designs. 


\section{References}

446 [1] R.J. Orth, T.J.B. Carruthers, W.C. Dennison, C.M. Duarte, J.W. Fourqurean, K.L.

447 Heck, A.R. Hughes, G.A. Kendrick, W.J. Kenworthy, S. Olyarnik, F.T. Short, M.

448 Waycott, S.L. Williams, A global crisis for seagrass ecosystems, Bioscience, 56 (2006)

449 987-996.

450 [2] M. Waycott, C.M. Duarte, T.J.B. Carruthers, R.J. Orth, W.C. Dennison, S. Olyarnik,

451 A. Calladine, J.W. Fourqurean, K.L. Heck, A.R. Hughes, G.A. Kendrick, W.J.

452 Kenworthy, F.T. Short, S.L. Williams, Accelerating loss of seagrasses across the globe

453 threatens coastal ecosystems, Proc. Natl. Acad. Sci. U. S. A., 106 (2009) 12377-12381.

454 [3] J.E. Duffy, Biodiversity and the functioning of seagrass ecosystems, Mar. Ecol.

455 Prog. Ser., 311 (2006) 233-250.

456 [4] IPCC, Climate Change 2007: The Physical Science Basis. Summary for

457 Policymakers., (2007) 1-21.

458 [5] S.I. Massa, S. Arnaud-Haond, G.A. Pearson, E.A. Serrao, Temperature tolerance

459 and survival of intertidal populations of the seagrass Zostera noltii (Hornemann) in

460 Southern Europe (Ria Formosa, Portugal), Hydrobiologia, 619 (2009) 195-201.

461 [6] R. Arya, M. Mallik, S.C. Lakhotia, Heat shock genes - integrating cell survival and

462 death, Journal of Biosciences, 32 (2007) 595-610.

463 [7] M.J. Schlesinger, Heat-Shock Proteins, J. Biol. Chem., 265 (1990) 12111-12114.

464 [8] S.J. Campbell, L.J. McKenzie, S.P. Kerville, Photosynthetic responses of seven

465 tropical seagrasses to elevated seawater temperature, J. Exp. Mar. Biol. Ecol., 330

466 (2006) 455-468.

467 [9] L.J. Cui, J.L. Li, Y.M. Fan, S. Xu, Z. Zhang, High temperature effects on

468 photosynthesis, PSII functionality and antioxidant activity of two Festuca arundinacea cultivars with different heat susceptibility, Botanical Studies, 47 (2006) 61-69.

[10] M. Almeselmani, P.S. Deshmukh, S. R.K., S.R. Kushwaha, T.P. Singh, Protective role of antioxidant enzymes under high temperature stress, Plant Science, 171 (2006) 382-388.

[11] J.J. Bull, M.R. Badgett, H.A. Wichman, J.P. Huelsenbeck, D.M. Hillis, A. Gulati, C. Ho, I.J. Molineux, Exceptional convergent evolution in a virus, Genetics, 147 (1997) 1497-1507.

[12] R. Woods, D. Schneider, C.L. Winkworth, M.A. Riley, R.E. Lenski, Tests of parallel molecular evolution in a long-term experiment with Escherichia coli, Proc. Natl. Acad. Sci. U. S. A., 103 (2006) 9107-9112.

479 [13] J. de Visser, A.D.L. Akkermans, R.F. Hoekstra, W.M. de Vos, Insertion-sequencemediated mutations isolated during adaptation to growth and starvation in Lactococcus lactis, Genetics, 168 (2004) 1145-1157. [14] S.E. Travers, Z.W. Tang, D. Caragea, K.A. Garrett, S.H. Hulbert, J.E. Leach, J.F. Bai, A. Saleh, A.K. Knapp, P.A. Fay, J. Nippert, P.S. Schnable, M.D. Smith, Variation in gene expression of Andropogon gerardii in response to altered environmental conditions associated with climate change, J. Ecol., 98 (2010) 374-383.

[15] H.H. Chou, M.H. Holmes, DNA sequence quality trimming and vector removal, Bioinformatics, 17 (2001) 1093-1104.

488 [16] G. Pertea, X.Q. Huang, F. Liang, V. Antonescu, R. Sultana, S. Karamycheva, Y.

489 Lee, J. White, F. Cheung, B. Parvizi, J. Tsai, J. Quackenbush, TIGR Gene Indices

490 clustering tools (TGICL): a software system for fast clustering of large EST datasets,

491 Bioinformatics, 19 (2003) 651-652. 

a universal tool for annotation, visualization and analysis in functional genomics research, Bioinformatics, 21 (2005) 3674-3676. [18] S. Gotz, J.M. Garcia-Gomez, J. Terol, T.D. Williams, S.H. Nagaraj, M.J. Nueda, M. Robles, M. Talon, J. Dopazo, A. Conesa, High-throughput functional annotation and data mining with the Blast2GO suite, Nucleic Acids Res., 36 (2008) 3420-3435. [19] C. Romualdi, S. Bortoluzzi, F. D'Alessi, G.A. Danieli, IDEG6: a web tool for detection of differentially expressed genes in multiple tag sampling experiments, Physiol. Genomics, 12 (2003) 159-162.

[20] S. Audic, J.M. Claverie, The significance of digital gene expression profiles, Genome Res., 7 (1997) 986-995.

[21] T.B.H. Reusch, A.S. Veron, C. Preuss, J. Weiner, L. Wissler, A. Beck, S. Klages, M. Kube, R. Reinhardt, E. Bornberg-Bauer, Comparative analysis of expressed sequence tag (EST) libraries in the seagrass Zostera marina subjected to temperature stress, Mar. Biotechnol., 10 (2008) 297-309. [22] L. Wissler, E. Dattolo, A.D. Moore, T.B.H. Reusch, J.L. Olsen, M. Migliaccio, E. Bornberg-Bauer, G. Procaccini, Dr. Zompo: an online data repository for Zostera marina and Posidonia oceanica ESTs, Database, (2009).

[23] B.C. Faircloth, MSATCOMMANDER: detection of microsatellite repeat arrays and automated, locus-specific primer design, Molecular Ecology Resources, 8 (2008) 92-94.

[24] X.J. Min, G. Butler, R. Storms, A. Tsang, OrfPredictor: predicting protein-coding regions in EST-derived sequences, Nucleic Acids Res., 33 (2005) W677-W680.

[25] P. Meinicke, UFO: a web server for ultra-fast functional profiling of whole genome protein sequences, BMC Genomics, 10 (2009).

[26] P.S. Krishna, Plant responses to heat stress, in: H. Hirt, K. Shinozaki (Eds.) Plant responses to abiotic stress, Springer-Verlag, Berlin Heidelberg, 2004, pp. 9-38.

[27] H.M. Beere, Death versus survival: functional interaction between the apoptotic and stress-inducible heat shock protein pathways, J. Clin. Investig., 115 (2005) 26332639.

[28] M.E. Cheetham, A.J. Caplan, Structure, function and evolution of DnaJ: conservation and adaptation of chaperone function, Cell Stress Chaperones, 3 (1998) 28-36.

[29] D.M. Cyr, T. Langer, M.G. Douglas, Dnaj-Like Proteins - Molecular Chaperones and Specific Regulators of Hsp70, Trends Biochem. Sci., 19 (1994) 176-181.

[30] G. Govind, H.V. ThammeGowda, P.J. Kalaiarasi, D.R. Iyer, S.K. Muthappa, S. Nese, U.K. Makarla, Identification and functional validation of a unique set of drought induced genes preferentially expressed in response to gradual water stress in peanut, Molecular Genetics and Genomics, 281 (2009) 591-605.

[31] S.G. Ahn, S.A. Kim, J.H. Yoon, P. Vacratsis, Heat-shock cognate 70 is required for the activation of heat-shock factor 1 in mammalian cells, Biochem. J., 392 (2005) 145152.

[32] P. Genevaux, C. Georgopoulos, W.L. Kelley, The Hsp70 chaperone machines of Escherichia coli: a paradigm for the repartition of chaperone functions, Mol. Microbiol., 66 (2007) 840-857.

[33] K. Aoki, F. Kragler, B. Xoconostle-Cazares, W.J. Lucas, A subclass of plant heat shock cognate 70 chaperones carries a motif that facilitates trafficking through plasmodesmata, Proc. Natl. Acad. Sci. U. S. A., 99 (2002) 16342-16347.

[34] C.M. Nicolet, E.A. Craig, Isolation and Characterization of Sti1, a Stress-Inducible Gene from Saccharomyces-Cerevisiae, Mol. Cell. Biol., 9 (1989) 3638-3646. 
[35] P.J. Ralph, Photosynthetic response of laboratory-cultured Halophila ovalis to thermal stress, Mar. Ecol. Prog. Ser., 171 (1998) 123-130. [36] C. Hutin, L. Nussaume, N. Moise, I. Moya, K. Kloppstech, M. Havaux, Early lightinduced proteins protect arabidopsis from photooxidative stress, Proc. Natl. Acad. Sci. U. S. A., 100 (2003) 4921-4926. [37] E.S. Johnson, B. Bartel, W. Seufert, A. Varshavsky, Ubiquitin as a Degradation Signal, Embo Journal, 11 (1992) 497-505. [38] A. Hershko, A. Ciechanover, The ubiquitin system, Annu. Rev. Biochem., 67 (1998) 425-479.

[39] L. Ferrat, C. Pergent-Martini, M. Romeo, Assessment of the use of biomarkers in aquatic plants for the evaluation of environmental quality: application to seagrasses, Aquatic Toxicology, 65 (2003) 187-204.

[40] A.J. Keys, I.F. Bird, M.J. Cornelius, P.J. Lea, R.M. Wallsgrove, B.J. Miflin, Photorespiratory Nitrogen Cycle, Nature, 275 (1978) 741-743.

[41] F. Vanlent, J.M. Verschuure, M.L.J. Vanveghel, Comparative-Study on Populations of Zostera-Marina L (Eelgrass) - in-Situ Nitrogen Enrichment and Light Manipulation, J. Exp. Mar. Biol. Ecol., 185 (1995) 55-76.

[42] K. Yoshida, P. Kaothien, T. Matsui, A. Kawaoka, A. Shinmyo, Molecular biology and application of plant peroxidase genes, Appl. Microbiol. Biotechnol., 60 (2003) 665670.

[43] N.C. Veitch, Horseradish peroxidase: a modern view of a classic enzyme, Phytochemistry, 65 (2004) 249-259.

[44] J.D. Hayes, D.J. Pulford, The glutathione S-Transferase supergene family: Regulation of GST and the contribution of the isoenzymes to cancer chemoprotection and drug resistance, Crit. Rev. Biochem. Mol. Biol., 30 (1995) 445-600.

[45] N.J. Robinson, A.M. Tommey, C. Kuske, P.J. Jackson, Plant Metallothioneins, Biochem. J., 295 (1993) 1-10.

[46] S. Moisyadi, J.I. Stiles, A Cdna-Encoding a Metallothionein I-Like Protein from Coffee Leaves (Coffea-Arabica), Plant Physiology, 107 (1995) 295-296.

[47] H. Nziengui, K. Bouhidel, D. Pillon, C. Der, F. Marty, B. Schoefs, Reticulon-like proteins in Arabidopsis thaliana: Structural organization and ER localization, FEBS Lett., 581 (2007) 3356-3362.

[48] F.X. Schmid, Protein-Folding - Prolyl Isomerases Join the Fold, Curr. Biol., 5 (1995) 993-994.

[49] M. Kruse, M. Brunke, A. Escher, A.A. Szalay, M. Tropschug, R. Zimmermann, Enzyme Assembly after De-Novo Synthesis in Rabbit Reticulocyte Lysate Involves Molecular Chaperones and Immunophilins, J. Biol. Chem., 270 (1995) 2588-2594. [50] S.F. Gothel, M.A. Marahiel, Peptidyl-prolyl cis-trans isomerases, a superfamily of ubiquitous folding catalysts, Cellular and Molecular Life Sciences, 55 (1999) 423-436. [51] S.C. Franca, P.G. Roberto, M.A. Marins, R.D. Puga, A. Rodrigues, J.O. Pereira, Biosynthesis of secondary metabolites in sugarcane, Genet. Mol. Biol., 24 (2001) 243250.

[52] S.L.A. Andrade, E.V. Patridge, J.G. Ferry, O. Einsle, Crystal structure of the NADH : Quinone oxidoreductase WrbA from Escherichia coli, J. Bacteriol., 189 (2007) 9101-9107. [53] R.D. Barber, D.W. Harmer, R.A. Coleman, B.J. Clark, GAPDH as a housekeeping gene: analysis of GAPDH mRNA expression in a panel of 72 human tissues, Physiol. Genomics, 21 (2005) 389-395. [54] N. Wedel, J. Soll, Evolutionary conserved light regulation of Calvin cycle activity by NADPH-mediated reversible phosphoribulokinase/CP12/glyceraldehyde-3- 
592 phosphate dehydrogenase complex dissociation, Proc. Natl. Acad. Sci. U. S. A., 95 593 (1998) 9699-9704.

594 [55] W.C. Plaxton, The organization and regulation of plant glycolysis, Annu. Rev. 595 Plant Physiol. Plant Mol. Biol., 47 (1996) 185-214.

596 [56] T. Mair, C. Warnke, K. Tsuji, S.C. Muller, Control of glycolytic oscillations by 597 temperature, Biophys. J., 88 (2005) 639-646.

598 [57] P.J. Ralph, Photosynthetic response of Halophila ovalis (R-Br.) Hook. f. to

599 combined environmental stress, Aquat. Bot., 65 (1999) 83-96.

600 [58] A. Bouck, T. Vision, The molecular ecologist's guide to expressed sequence tags, 601 Mol. Ecol., 16 (2007) 907-924.

602

603

604

605 

min, 2h, 4h and 24h) and the control (blank).

\begin{tabular}{|l|l|l|l|l|l|}
\hline Library & $\mathbf{3 0} \mathbf{m i n}$ & $\mathbf{2 h}$ & $\mathbf{4 h}$ & $\mathbf{2 4 h}$ & control \\
\hline Total number of clones sequenced & 1,724 & 1,795 & 1,756 & 1,734 & 1,585 \\
\hline Number of high quality sequences & $\begin{array}{l}1,657 \\
(96.1 \%)\end{array}$ & $\begin{array}{l}1,698 \\
(94.6 \%)\end{array}$ & $\begin{array}{l}1,553 \\
(88.4 \%)\end{array}$ & $\begin{array}{l}1,563 \\
(90.1 \%)\end{array}$ & $\begin{array}{l}1,328 \\
(83.8 \%)\end{array}$ \\
\hline Total number of successful sequences & $\begin{array}{l}1,635 \\
(94.8 \%)\end{array}$ & $\begin{array}{l}1,671 \\
(93.1 \%)\end{array}$ & $\begin{array}{l}1,536 \\
(87.5 \%)\end{array}$ & $\begin{array}{l}1,535 \\
(88.5 \%)\end{array}$ & $\begin{array}{l}1,309 \\
(82.6 \%)\end{array}$ \\
\hline Number of contigs & 170 & 174 & 151 & 145 & 145 \\
\hline Number of clones included in the contigs & $\begin{array}{l}733 \\
(44.8 \%)\end{array}$ & $\begin{array}{l}700 \\
(41.9 \%)\end{array}$ & $\begin{array}{l}479 \\
(31.2 \%)\end{array}$ & $\begin{array}{l}439 \\
(28.6 \%)\end{array}$ & $\begin{array}{l}511 \\
(39.0 \%)\end{array}$ \\
\hline Average contig length & $755 \mathrm{bp}$ & $783 \mathrm{bp}$ & $737 \mathrm{bp}$ & $736 \mathrm{bp}$ & $682 \mathrm{bp}$ \\
\hline Number of singletons & $\begin{array}{l}902 \\
(55.2 \%)\end{array}$ & $\begin{array}{l}971 \\
(58.1 \%)\end{array}$ & $\begin{array}{l}1,057 \\
(68.8 \%)\end{array}$ & $\begin{array}{l}1,096 \\
(71.4 \%)\end{array}$ & $\begin{array}{l}798 \\
(61.0 \%)\end{array}$ \\
\hline Tentative UniGenes (TUGs) \\
(a+b) & 1,072 & 1,145 & 1,208 & 1,241 & 943 \\
\hline Number of TUG with no ORF & $\begin{array}{l}2 \\
(0.18 \%)\end{array}$ & $\begin{array}{l}4 \\
(0.35 \%)\end{array}$ & $\begin{array}{l}1 \\
(0.08 \%)\end{array}$ & $\begin{array}{l}1 \\
(0.08 \%)\end{array}$ & $\begin{array}{l}13 \\
(1.38 \%)\end{array}$ \\
\hline Number of TUGs with a successful blast & $\begin{array}{l}907 \\
(84.6 \%)\end{array}$ & $\begin{array}{l}985 \\
(86.0 \%)\end{array}$ & $\begin{array}{l}1,030 \\
(85.3 \%)\end{array}$ & $\begin{array}{l}1,031 \\
(83.1 \%)\end{array}$ & $\begin{array}{l}690 \\
(73.2 \%)\end{array}$ \\
\hline Number of successfully mapped TUGs & $\begin{array}{l}745 \\
(69.5 \%)\end{array}$ & $\begin{array}{l}824 \\
(72.0 \%)\end{array}$ & $\begin{array}{l}832 \\
(68.9 \%)\end{array}$ & $\begin{array}{l}914 \\
(73.7 \%)\end{array}$ & $\begin{array}{l}628 \\
(66.6 \%)\end{array}$ \\
\hline Number of successfully annotated TUGs & $\begin{array}{l}600 \\
(56.0 \%)\end{array}$ & $\begin{array}{l}639 \\
(55.8 \%)\end{array}$ & $\begin{array}{l}650 \\
(53.8 \%)\end{array}$ & $\begin{array}{l}730 \\
(58.8 \%)\end{array}$ & $\begin{array}{l}527 \\
(55.9 \%)\end{array}$ \\
\hline
\end{tabular}


609 Table 2: Main protein families. All families with at least 10 sequence reads in one of 610 the libraries.

\begin{tabular}{|c|c|c|c|c|c|}
\hline & $30 \mathrm{~min}$ & $2 \mathrm{~h}$ & $4 \mathrm{~h}$ & $24 \mathrm{~h}$ & control \\
\hline (Hsp70 protein) & 28 & 31 & 16 & 13 & 1 \\
\hline (Protein kinase domain) & 16 & 23 & 19 & 20 & 12 \\
\hline (Chlorophyll A-B binding protein) & 16 & 15 & 16 & 15 & 16 \\
\hline $\begin{array}{l}\text { (RNA recognition motif. (a.k.a. } \\
\text { RRM, RBD, or RNP domain)) }\end{array}$ & 11 & 8 & 8 & 8 & 5 \\
\hline (Cytochrome P450) & 6 & 2 & 10 & 2 & 4 \\
\hline
\end{tabular}

611 
612 Table 3: EST-SSRs found in all TUGs.

\begin{tabular}{|c|c|c|c|c|c|c|c|c|c|c|c|c|}
\hline \multirow[b]{2}{*}{ Motif } & \multicolumn{12}{|c|}{ Number of repeat units } \\
\hline & 6 & 7 & 8 & 9 & 10 & 11 & 12 & 13 & 14 & 15 & 16 & Total \\
\hline $\mathrm{AC}$ & & 2 & 1 & & & & & & & & & 3 \\
\hline AG & 6 & 2 & 2 & 1 & & 1 & 1 & 1 & 1 & 1 & 1 & 17 \\
\hline AT & 8 & 7 & 2 & 1 & 1 & 2 & & 1 & & 1 & & 23 \\
\hline $\mathrm{AAC}$ & 1 & & & & & & & & & & & 1 \\
\hline AAG & 7 & 8 & 1 & 1 & & & 1 & 1 & & & & 19 \\
\hline AAT & 4 & & & & & & & & & & & 4 \\
\hline $\mathrm{ACC}$ & & 1 & 1 & & & & & & & & & 2 \\
\hline ACT & 1 & & & & & & & & & & & 1 \\
\hline $\mathrm{AGC}$ & 1 & & & & & & & & & & & 1 \\
\hline AGG & 2 & 2 & & & & & & & & & & 4 \\
\hline ATC & 1 & 1 & & & & & & & & & & 2 \\
\hline $\mathrm{CCG}$ & 2 & & & & & & & & & & & 2 \\
\hline AATG & & & & & 1 & & & & & & & 1 \\
\hline AATT & & 1 & & & & & & & & & & 1 \\
\hline AGAT & 1 & & & & & & & & & & & 1 \\
\hline ATCC & & 1 & & & & & & & & & & 1 \\
\hline AACATG & 1 & & & & & & & & & & & 1 \\
\hline AATCAC & 1 & & & & & & & & & & & 1 \\
\hline AGATGG & 1 & & & & & & & & & & & 1 \\
\hline Total & 31 & 19 & 7 & 3 & 1 & 1 & 2 & 3 & 1 & 2 & 1 & 86 \\
\hline
\end{tabular}




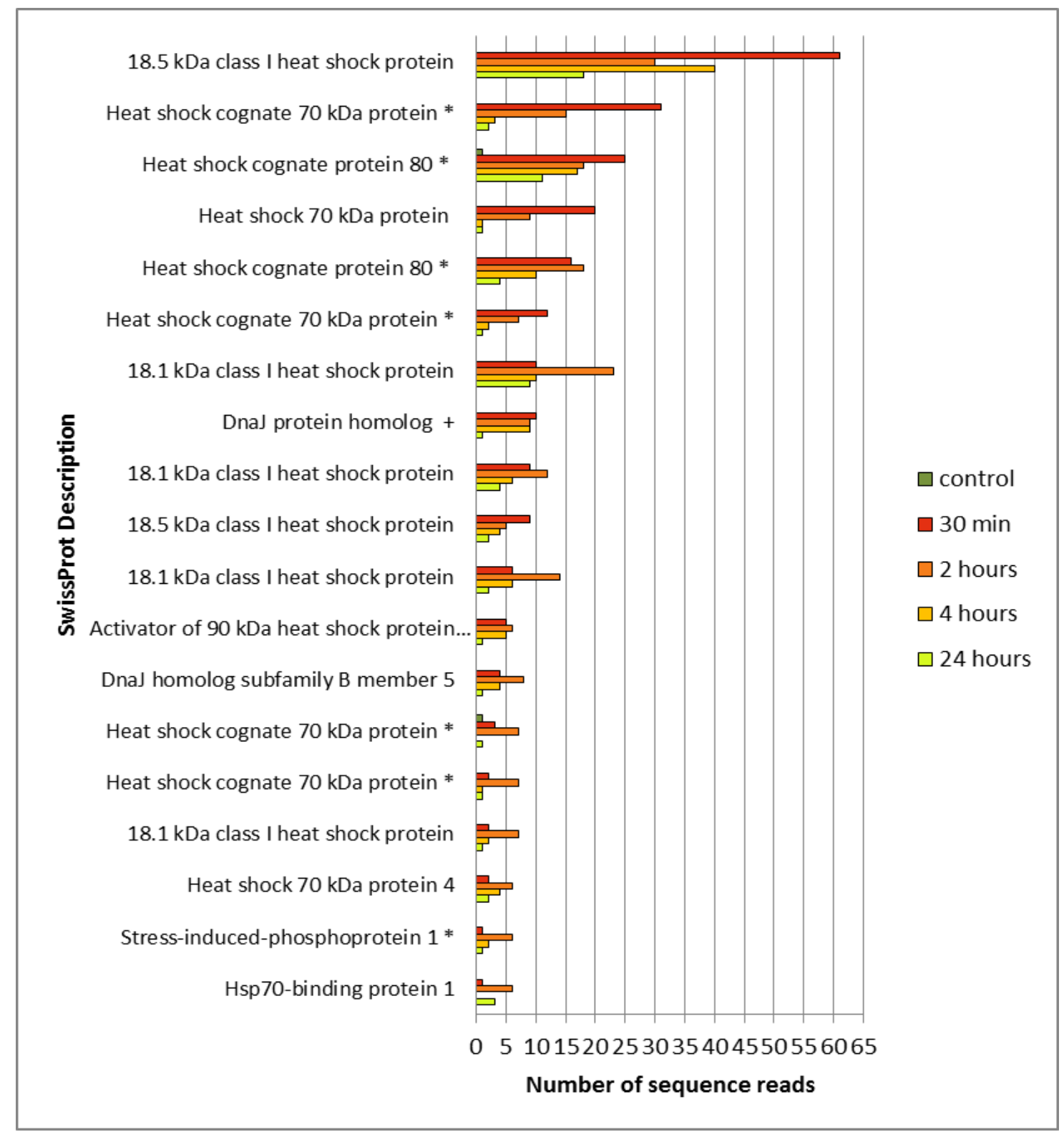

\section{Figure 1: Number of sequence reads of each 'molecular chaperone' TUG with}

616 significantly different expressions in each library. SwissProt descriptions were used

617 to identify each TUG. The number of sequence reads for the control library is shown in

618 white, stress libraries are as follows: 30 minutes in black, $2 \mathrm{~h}$ in dark grey, $4 \mathrm{~h}$ in medium

619 grey and 24h in light grey. It is also shown when a corresponding hit was found in Dr.

620 Zompo database for Zostera marina $\left(^{*}\right)$ and Posidonia oceanica (+). 


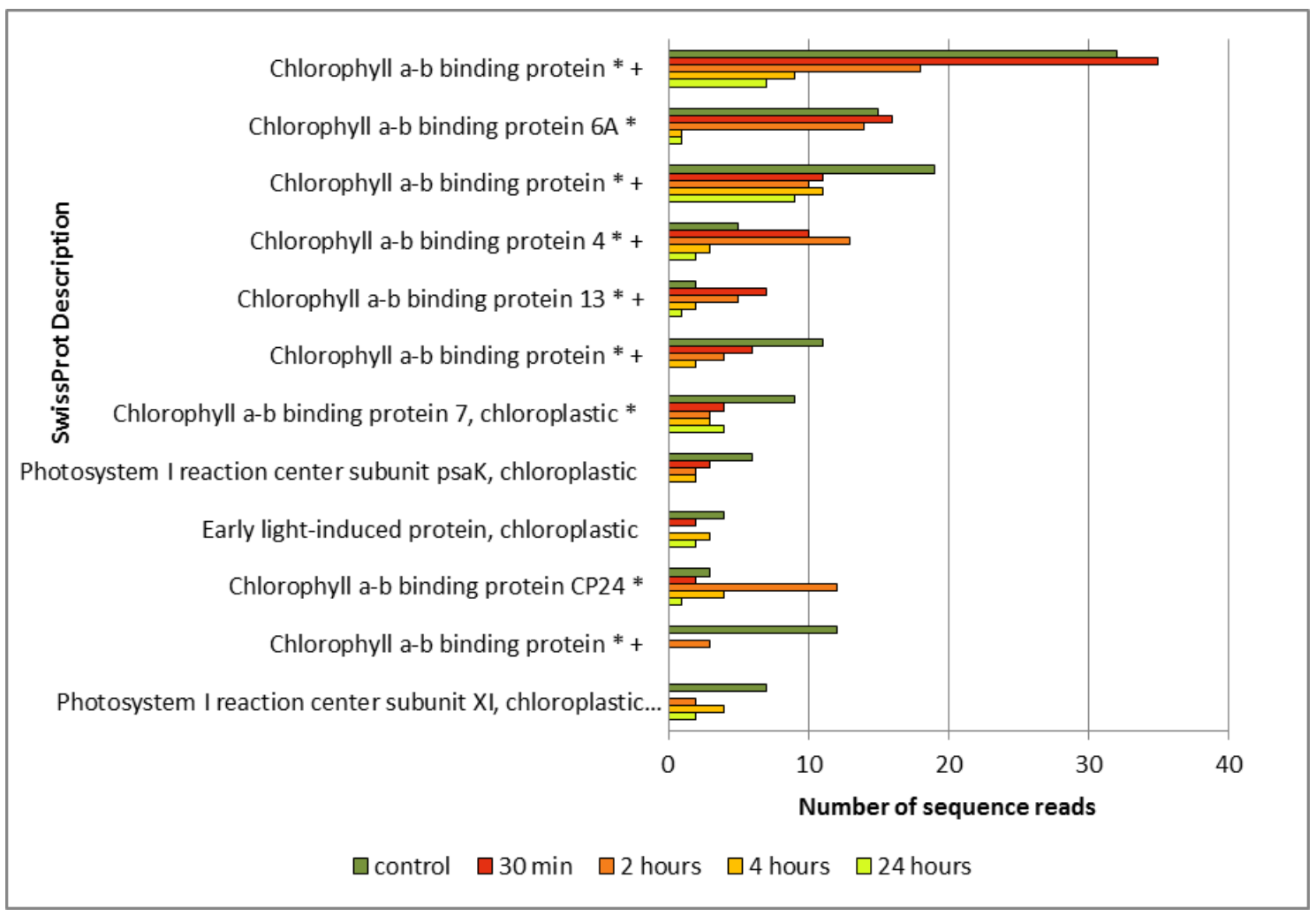

622 Figure 2: Number of sequence reads of each photosynthesis-related TUG with

623 significantly different expressions in each library. SwissProt descriptions were used

624 to identify each TUG. The number of sequence reads for the control library is shown in

625 white, stress libraries are as follows: 30 minutes in black, $2 \mathrm{~h}$ in dark grey, $4 \mathrm{~h}$ in medium

626 grey and $24 \mathrm{~h}$ in light grey. It is also shown when a corresponding hit was found in Dr.

627 Zompo database for Zostera marina $(*)$ and Posidonia oceanica $(+)$. 


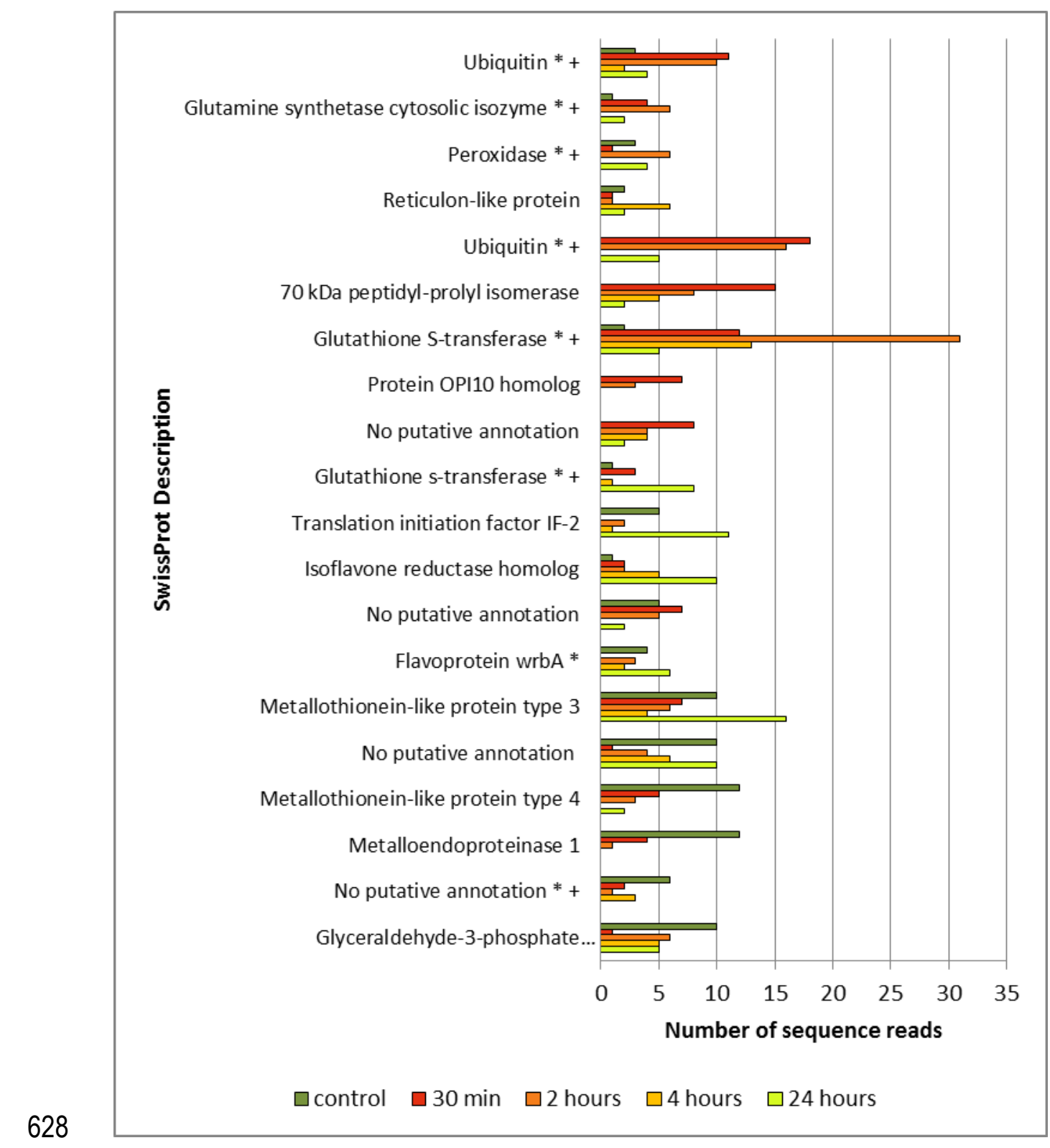

629 Figure 3: Number of sequence reads of other TUGs of interest with significantly

630 different expressions in each library. SwissProt descriptions were used to identify

631 each TUG. The number of sequence reads for the control library is shown in white,

632 stress libraries are as follows: 30 minutes in black, $2 \mathrm{~h}$ in dark grey, $4 \mathrm{~h}$ in medium grey

633 and $24 \mathrm{~h}$ in light grey. It is also shown when a corresponding hit was found in Dr.

634 Zompo database for Zostera marina $\left(^{*}\right)$ and Posidonia oceanica (+). 